\title{
Limited Model Information Control Design for Linear Discrete-Time Systems with Stochastic Parameters
}

\author{
Farhad Farokhi and Karl H. Johansson
}

\begin{abstract}
We design optimal local controllers for interconnected discrete-time linear systems with stochastically varying parameters using exact local model information and statistical beliefs about the model of the rest of the system. We study the value of model information in control design using the closedloop performance degradation caused by the lack of full model information in the control design procedure. This performance degradation is captured using the ratio of the cost of the optimal controller with limited model information over the cost of the optimal controller with full model information. Both finitehorizon and infinite-horizon cost functions are considered. A numerical example illustrates the developed approach.
\end{abstract}

\section{INTRODUCTION}

Large-scale networked systems, such as automated highways, power grid, and other shared infrastructure [1]-[3], have attracted much attention recently. These systems are typically composed of several locally controlled subsystems connected to each other. In designing the local controllers, it is often assumed that the complete model of the system is available. However, this assumption is usually not easily satisfied in practice. For instance, consider power grid control problem with power generated in power generators and distributed throughout the network via transmission lines. It is common to assume that the loads' power consumption in such a network are modeled stochastically with a priori known statistics (i.e., mean and variance) [4]-[6]. When the load variations are "small enough", local generators meet these demand variations. These variations shift the generators operating points, and consequently, change their model parameters. As power networks are typically implemented over a vast geographical area, it is inefficient or even impossible to gather all these model information variations or to identify all the parameters globally at one place. This motivates our interest in designing local controllers for these system based on only local model information and statistical model information of (i.e., our beliefs about) other components. Similar reasoning can also be made for process control, intelligent transportation, and water distribution systems [7].

The main contribution of this paper is to study limited model information control design for discrete-time linear systems with stochastically varying parameters. Recently, there have been studies in optimal control design for discretetime linear time-invariant systems using limited model information [7]-[10]. However, in these studies, the model

F. Farokhi and K. H. Johansson are with ACCESS Linnaeus Center, School of Electrical Engineering, KTH-Royal Institute of Technology, SE10044 Stockholm, Sweden. E-mails: \{farokhi,kallej\} @ee.kth.se

The work was supported by the Swedish Research Council and the Knut and Alice Wallenberg Foundation. information of other subsystems are completely unknown which typically results in conservative controllers. This forces the designer to study the worst-case behavior of the control design methods. In this paper, we take a new approach by assuming that a statistical model is available for other subsystems' parameters. There have been many studies in optimal control design for linear discrete-time systems with stochastic parameters [11]-[15]. In these papers, the optimal controller is typically calculated as a function of only model parameter statistics. Contrary to those works, in this paper, we assume each subcontroller design is done using its own perfect model information and other subsystems model statistics. Using the closed-loop performance of this optimal controller, we study the effect of the lack of full model information on the quality of the controllers that one could design using only local model information accompanied with statistical behavior of the other subsystems. Specifically, we study the ratio of the cost of the optimal control design strategy with limited model information over the cost of the optimal control design strategy with full model information. It worth mentioning that, in this paper, we focus on full state-feedback controllers. This assumption can be justified by high-bandwidth wireless communication, which might enable us to transmit all sensor measurements across the networked system. However, the global model information might still be unavailable since identifying the parameters globally in a systems is hard. As a future direction for research, one might be able to generalize the results of this paper for designing structured state-feedback controllers following the same reasoning as in [16]. Lastly, we demonstrate the optimal controller with limited model information and full model information on a numerical example, and compare them with previous results of optimal control design with statistical model information [11]. In this example, one can easily see the dependencies of the different subcontrollers to exact model parameters and their statistics.

The rest of the paper is organized as follows. We start by introducing the system model in Section II. In Section III, we design optimal controller for each subsystem using its own model information and the statistical properties of other subsystems. This is done, at first, considering finite-horizon optimal control problem. Then, we generalize these results to infinite-horizon cost functions. In Section IV, we study the value of information in optimal control design using the ratio of the cost of the optimal controller with limited model information to the cost of the optimal controller strategy with full model information. We illustrate the results of the paper on a numerical example in Section V. Finally, the 
conclusions and directions for future research are presented in Section VI.

\section{A. Notation}

Matrices are denoted by capital roman letters such as $A$. $A_{i j}$ denotes a submatrix of matrix $A$, the dimension and the position of which will be defined in the text. The entry in the $i^{\text {th }}$ row and the $j^{\text {th }}$ column of the matrix $A$ is $a_{i j}$.

Let $S_{++}^{n}\left(S_{+}^{n}\right)$ be the set of symmetric positive definite (positive semidefinite) matrices in $\mathbb{R}^{n \times n}$. $A>(\geq) 0$ means $A \in S_{++}^{n}\left(S_{+}^{n}\right)$ and $A>(\geq) B$ means $A-B>(\geq) 0$.

Let $A \otimes B \in \mathbb{R}^{n p \times q m}$ denote the Kronecker product between matrices $A \in \mathbb{R}^{n \times m}$ and $B \in \mathbb{R}^{p \times q}$; i.e.,

$$
A \otimes B=\left[\begin{array}{ccc}
a_{11} B & \cdots & a_{1 m} B \\
\vdots & \ddots & \vdots \\
a_{n 1} B & \cdots & a_{n m} B
\end{array}\right]
$$

For any positive integers $n$ and $m$, we define the mapping vec $: \mathbb{R}^{n \times m} \rightarrow \mathbb{R}^{n m}$ as

$$
\operatorname{vec}(A)=\left[\begin{array}{llll}
A_{1}^{\top} & A_{2}^{\top} & \ldots & A_{m}^{\top}
\end{array}\right]^{\top},
$$

where $A_{i}$, for all $1 \leq i \leq m$, are the columns of matrix $A \in \mathbb{R}^{n \times m}$. The mapping vec ${ }^{-1}: \mathbb{R}^{n m} \rightarrow \mathbb{R}^{n \times m}$ means the inverse of the operator $\operatorname{vec}(\cdot)$.

For any given positive integers $n$ and $m$, and compatible matrices $A \in \mathbb{R}^{n \times n}, P \in \mathbb{R}^{n \times n}, B \in \mathbb{R}^{n \times m}$, and $R \in$ $\mathbb{R}^{m \times m}$, we define the discrete Riccati operator

$$
\mathcal{R}(A, P, B, R)=A^{\top}\left(P-P B\left(R+B^{\top} P B\right)^{-1} B^{\top} P\right) A .
$$

\section{Stochastic Parameter Systems}

Consider a discrete-time linear control system with stochastically varying parameters composed of $N$ subsystems with each subsystem represented in state-space form as

$$
x_{i}(k+1)=\sum_{j=1}^{N} A_{i j}(k) x_{j}(k)+B_{i i}(k) u_{i}(k),
$$

where, for each $1 \leq i \leq N, x_{i}(k) \in \mathbb{R}^{n_{i}}$ and $u_{i}(k) \in \mathbb{R}^{m_{i}}$ are subsystem $i$ state vector and control input, respectively.

We assume that the submatrices $A_{i j}(k)$, for all $1 \leq i, j \leq$ $N$, are independent and identically distributed stochastic processes in time. Therefore, $A_{i j}\left(k_{1}\right) \perp A_{i j}\left(k_{2}\right)$ for all $1 \leq i, j \leq N$ and $k_{1} \neq k_{2}$, where the operator $\perp$ represents the stochastic independence of two random variables. Systems with stochastically varying parameters have been studied in, for instance, the area of power systems [4], [5] and process control [17]. System theoretic properties and various control design methods have been developed for such systems [11]-[14]. Furthermore, we assume that the subsystems are stochastically independent of each other. Consequently, $A_{i j}(k) \perp A_{i^{\prime} j^{\prime}}(k)$ for all $1 \leq j, j^{\prime} \leq N$ and $1 \leq i \neq i^{\prime} \leq N$. We will use the notations

$$
\bar{A}_{i j}(k)=\mathbb{E}\left\{A_{i j}(k)\right\}, \quad \tilde{A}_{i j}(k)=A_{i j}(k)-\bar{A}_{i j}(k) .
$$

Let us augment the state vectors of all the subsystem into

$$
x(k)=\left[\begin{array}{c}
x_{1}(k) \\
\vdots \\
x_{N}(k)
\end{array}\right] \in \mathbb{R}^{n}, \quad u(k)=\left[\begin{array}{c}
u_{1}(k) \\
\vdots \\
u_{N}(k)
\end{array}\right] \in \mathbb{R}^{m},
$$

with $n=\sum_{i=1}^{N} n_{i}$ and $m=\sum_{i=1}^{N} m_{i}$, and get the global state-space description of the system as

$$
x(k+1)=A(k) x(k)+B(k) u(k) .
$$

We use the notations

$$
\bar{A}(k)=\mathbb{E}\{A(k)\}, \quad \tilde{A}(k)=A(k)-\bar{A}(k) .
$$

In order to simplify later calculations, for all $1 \leq i \leq N$, we further introduce the notations

$$
B_{i}(k)=\left[\begin{array}{c}
0 \\
\vdots \\
0 \\
B_{i i}(k) \\
0 \\
\vdots \\
0
\end{array}\right], \quad \tilde{A}_{i}(k)=\left[\begin{array}{ccc}
0 & \cdots & 0 \\
\vdots & \ddots & \vdots \\
0 & \cdots & 0 \\
\tilde{A}_{i 1}(k) & \cdots & \tilde{A}_{i N}(k) \\
0 & \cdots & 0 \\
\vdots & \ddots & \vdots \\
0 & \cdots & 0
\end{array}\right] .
$$

\section{Optimal Control Design with Limited Model}

\section{INFORMATION}

In this section, we study the finite-horizon and infinitehorizon optimal control design using exact local model information and statistical beliefs about other subsystems. Therefore, when designing subcontroller $i$, we can only observe the first $\mathbb{E}\{A(k)\}$ and second order $\mathbb{E}\{\tilde{A}(k) \otimes \tilde{A}(k)\}$ moments of the global system model, together with the exact local model information $\left\{A_{i j}(k) \mid 1 \leq j \leq N\right\}$. We start with minimizing the finite-horizon cost function in next subsection.

\section{A. Finite-Horizon Cost Function}

In the finite-horizon optimal control design problem, we minimize the average performance criterion

$$
\begin{aligned}
J_{T}\left(x_{0},\{u(k)\}_{k=0}^{T-1}\right)=\mathbb{E}\left\{x(T)^{\top} Q(T) x(T)\right. \\
\left.+\sum_{k=0}^{T-1} x(k)^{\top} Q(k) x(k)+u(k)^{\top} R(k) u(k)\right\},
\end{aligned}
$$

subject to the system dynamics in (1) and the model information constraints described above. In (2), we assume that $Q(k) \geq 0$ for all $0 \leq k \leq T$ and $R(k)>0$ for all $0 \leq k \leq T-1$. The next theorem presents the solution of this finite-horizon optimal control problem.

THEOREM 3.1: The solution of the finite horizon optimal control problem is given in (3), where the sequence of matrices $\{P(k)\}_{k=0}^{T}$ can be calculated using the backward difference equation

$$
\begin{aligned}
P(k)= & Q(k)+\mathcal{R}(\bar{A}(k), P(k+1), B(k), R) \\
& +\sum_{i=1}^{N} \mathbb{E}\left\{\mathcal{R}\left(\tilde{A}_{i}(k), P(k+1), B_{i}(k), R_{i i}\right)\right\},
\end{aligned}
$$




$$
\begin{aligned}
u(k)= & -\left(R(k)+B(k)^{\top} P(k+1) B(k)\right)^{-1} B(k)^{\top} P(k+1) \bar{A}(k) x(k) \\
& -\left[\begin{array}{c}
\left(R_{11}(k)+B_{1}(k)^{\top} P(k+1) B_{1}(k)\right)^{-1} B_{1}(k)^{\top} P(k+1) \tilde{A}_{1}(k) \\
\vdots \\
\left(R_{N N}(k)+B_{N}(k)^{\top} P(k+1) B_{N}(k)\right)^{-1} B_{N}(k)^{\top} P(k+1) \tilde{A}_{N}(k)
\end{array}\right] x(k),
\end{aligned}
$$

with the boundary condition $P(T)=Q(T)$.

Proof: We can solve the finite-horizon optimal control problem using dynamic programming

$$
\begin{gathered}
V_{k}(x(k))=\inf _{u(k)} \mathbb{E}\left\{x(k)^{\top} Q(k) x(k)+u(k)^{\top} R(k) u(k)\right. \\
\left.+V_{k+1}(A(k) x(k)+B(k) u(k))\right\},
\end{gathered}
$$

where $V_{T}(x(T))=x(T)^{\top} Q(T) x(T)$. Let us assume, for all $k$, that

$$
V_{k}(x(k))=x(k)^{\top} P(k) x(k)
$$

where $P(k) \in \mathcal{S}_{+}^{n}$. This is without loss of generality since $V_{T}(x(T))=x(T)^{\top} Q(T) x(T)$ is a quadratic function of the state vector $x(T)$ and, using dynamic programming as it is shown in the rest of the proof, $V_{k}(x(k))$ remains a quadratic function of $x(k)$ if $V_{k+1}(x(k+1))$ is a quadratic function of $x(k+1)$. To minimize the running cost (5), in each timestep $k$, we solve

$$
\begin{aligned}
\frac{\partial}{\partial u_{i}(k)} \mathbb{E}\left\{x(k)^{\top} Q(k) x(k)+u(k)^{\top} R(k) u(k)\right. \\
\quad+(A(k) x(k)+B(k) u(k))^{\top} \\
\quad \times P(k+1)(A(k) x(k)+B(k) u(k))\}=0,
\end{aligned}
$$

for all $1 \leq i \leq N$. For simplicity of calculations, we split the control signal into two parts as $u_{i}(k)=\bar{u}_{i}(k)+\tilde{u}_{i}(k)$ where $\bar{u}_{i}(k)$ is only a function of plant model parameter statistics (i.e., mean and variance) and $\tilde{u}_{i}(k)$ is a function of the exact observation of subsystem $i$ model parameters (which is only available in subcontroller $i$ ). Thus, for the first part, we get

$$
R(k) \bar{u}(k)+B(k)^{\top} P(k+1)(\bar{A}(k) x(k)+B(k) \bar{u}(k))=0,
$$

where $\bar{u}(k)=\left[\begin{array}{lll}\bar{u}_{1}(k)^{T} & \cdots & \bar{u}_{N}(k)^{T}\end{array}\right]^{T}$. This results in

$$
\begin{aligned}
\bar{u}(k)=-\left(R(k)+B(k)^{\top} P(k+1) B(k)\right)^{-1} & \\
& \times B(k)^{\top} P(k+1) \bar{A}(k) x(k) .
\end{aligned}
$$

Now, by substituting $\bar{u}(k)$ from (7) inside (6), and taking expectation over model parameters of all subsystems $j \neq i$, we get

$\mathbb{E}\left\{2 R_{i i}(k) \tilde{u}_{i}(k)+2 B_{i}^{\top} P(k+1)\left(\tilde{A}_{i}(k) x(k)+B_{i} \tilde{u}_{i}(k)\right)\right\}=0$,

and as a result

$$
\begin{aligned}
\tilde{u}_{i}(k)=-\left(R_{i i}(k)+\right. & \left.B_{i}(k)^{\top} P(k+1) B_{i}(k)\right)^{-1} \\
& \times B_{i}(k)^{\top} P(k+1) \tilde{A}_{i}(k) x(k) .
\end{aligned}
$$

By substituting the optimal controller from (7)-(8) inside the recursive cost equation in (5), we get the cost function update equation in (9) where

$$
\begin{aligned}
\bar{K}(k)=-\left(R(k)+B(k)^{\top}\right. & P(k+1) B(k))^{-1} \\
& \times B(k)^{\top} P(k+1) \bar{A}(k),
\end{aligned}
$$

and

$$
\begin{aligned}
\tilde{K}_{i}(k)=-\left(R_{i i}(k)+B_{i}(k)^{\top}\right. & \left.P(k+1) B_{i}(k)\right)^{-1} \\
& \times B_{i}(k)^{\top} P(k+1) \tilde{A}_{i}(k) .
\end{aligned}
$$

One can simplify (9) into (10) by expanding and reordering its terms. Now, since the equality in (10) is true irrespective of the value of the state vector $x(k)$, we get the recurrence relation in (4). This concludes the proof.

Note that the optimal controller is composed of two parts: the first part is only a function of the parameter statistics while the second part is a function of exact local model parameters.

It is interesting to note that the optimal controller does not assume any special probability distribution on the model parameters. The designer only need to know the first and second moments of the parameters.

REMARK 3.1 ([11]): It might seem computationally difficult to calculate $\mathbb{E}\left\{\tilde{A}_{i}(k)^{\top} Z(k) \tilde{A}_{i}(k)\right\}$ for each time-step $k$ and any given matrix $Z(k)$. However, it suffices to calculate $\mathbb{E}\left\{\tilde{A}_{i}(k)^{\top} \otimes \tilde{A}_{i}(k)\right\}$ once, and then use the identity

$$
\begin{aligned}
\mathbb{E}\left\{\tilde{A}_{i}(k)^{\top} Z(k) \tilde{A}_{i}(k)\right\} & \\
& =\operatorname{vec}^{-1}\left(\mathbb{E}\left\{\left(\tilde{A}_{i}(k) \otimes \tilde{A}_{i}(k)\right)^{\top} \operatorname{vec}(Z(k))\right\}\right) \\
& =\operatorname{vec}^{-1}\left(\mathbb{E}\left\{\tilde{A}_{i}(k) \otimes \tilde{A}_{i}(k)\right\}^{\top} \operatorname{vec}(Z(k))\right) .
\end{aligned}
$$

With this result in hand, we are ready for solving the infinite-horizon optimal control problem.

\section{B. Infinite-Horizon Cost Function}

In this subsection, we use the results proved in the previous subsection to minimize the infinite-horizon average performance criterion

$$
J_{\infty}\left(x_{0},\{u(k)\}_{k=0}^{\infty}\right)=\lim _{T \rightarrow \infty} J_{T}\left(x_{0},\{u(k)\}_{k=0}^{T-1}\right),
$$

with $Q(k)=Q>0$ and $R(k)=R>0$ for all $0 \leq k \leq$ $T-1$ and $Q(T)=0$.

AsSUMPTION 3.1: For all $k$, the model parameters of the system in (1) satisfy

- $\bar{A}(k)=\bar{A} \in \mathbb{R}^{n \times n}$ and $\mathbb{E}\{A(k) \otimes A(k)\}=\Sigma \in \mathbb{R}^{n^{2} \times n^{2}}$; - $B(k)=B \in \mathbb{R}^{n \times m}$.

We borrow the following technical definition and assumption from [11] to prove the results of this subsection. We refer interested readers to [11] for numerical methods of checking this condition.

DEFINITION 3.1: A discrete-time linear with stochastically varying parameters of the form (1) is called mean square stabilizable if there exists a matrix $L \in \mathbb{R}^{m \times n}$ such that the closed-loop system with controller $u(k)=L x(k)$ is mean square stable; i.e., $\lim _{k \rightarrow+\infty} \mathbb{E}\left\{x(k)^{\top} x(k)\right\}=0$. 


$$
\begin{aligned}
x(k)^{\top} & P(k) x(k) \\
= & x(k)^{\top} Q(k) x(k)+x(k)^{\top}\left\{\bar{K}(k)^{\top} R(k) \bar{K}(k)+(\bar{A}(k)+B(k) \bar{K}(k))^{\top} P(k+1)(\bar{A}(k)+B(k) \bar{K}(k))\right\} x(k) \\
& +\sum_{i=1}^{N} x(k)^{\top} \mathbb{E}\left\{\tilde{K}_{i}(k)^{\top} R_{i i}(k) \tilde{K}_{i}(k)+\left(\tilde{A}_{i}(k)+B_{i}(k) \tilde{K}_{i}(k)\right)^{\top} P(k+1)\left(\tilde{A}_{i}(k)+B_{i}(k) \tilde{K}_{i}(k)\right)\right\} x(k),
\end{aligned}
$$

$$
\begin{aligned}
x( & (k)^{\top} P(k) x(k) \\
= & x(k)^{\top} Q(k) x(k)+x(k)^{\top} \bar{A}(k)^{\top}\left(P(k+1)-P(k+1) B(k)\left(R+B(k)^{\top} P(k+1) B(k)\right)^{-1} B(k)^{\top} P(k+1)\right) \bar{A}(k) x(k) \\
& +\sum_{i=1}^{N} x(k)^{\top} \mathbb{E}\left\{\tilde{A}_{i}(k)^{\top}\left(P(k+1)-P(k+1) B_{i}(k)\left(R_{i i}(k)+B_{i}(k)^{\top} P(k+1) B_{i}(k)\right)^{-1} B_{i}(k)^{\top} P(k+1)\right) \tilde{A}_{i}(k)\right\} x(k) \\
= & x(k)^{\top} Q(k) x(k)+x(k)^{\top} \mathcal{R}(\bar{A}(k), P(k+1), B(k), R) x(k)+\sum_{i=1}^{N} x(k)^{\top} \mathbb{E}\left\{\mathcal{R}\left(\tilde{A}_{i}(k), P(k+1), B_{i}(k), R_{i i}\right)\right\} x(k) .
\end{aligned}
$$

THEOREM 3.2: Assume that the discrete-time linear stochastic system given in (1) satisfies Assumption 3.1 and is mean square stabilizable. The solution of the infinite-horizon optimal control problem is given by

$$
\begin{aligned}
u(k)= & -\left(R+B^{\top} P B\right)^{-1} B^{\top} P \bar{A} x(k) \\
& -\left[\begin{array}{c}
\left(R_{11}+B_{1}^{\top} P B_{1}\right)^{-1} B_{1}^{\top} P \tilde{A}_{1}(k) \\
\vdots \\
\left(R_{N N}+B_{N}^{\top} P B_{N}\right)^{-1} B_{N}^{\top} P \tilde{A}_{N}(k)
\end{array}\right] x(k),
\end{aligned}
$$

where $P$ is the unique finite positive-definite solution of the modified discrete algebraic Riccati equation

$$
P=Q+\mathcal{R}(\bar{A}, P, B, R)+\sum_{i=1}^{N} \mathbb{E}\left\{\mathcal{R}\left(\tilde{A}_{i}(k), P, B_{i}, R_{i i}\right)\right\} .
$$

Furthermore, this controller mean square stabilizes the system and

$$
\inf _{\{u(k)\}_{k=0}^{\infty}} J_{\infty}\left(x_{0},\{u(k)\}_{k=0}^{\infty}\right)=x_{0}^{\top} P x_{0} .
$$

Proof: First, let us define the mapping $f: \mathcal{S}_{+}^{n} \rightarrow \mathcal{S}_{+}^{n}$ such that, for any $X \in \mathcal{S}_{+}^{n}$, we get

$$
\begin{aligned}
& f(X)=Q+\bar{A}^{\top}\left(X-X B\left(R+B^{\top} X B\right)^{-1} B^{\top} X\right) \bar{A} \\
& +\sum_{i=1}^{N} \mathbb{E}\left\{\tilde{A}_{i}^{\top}\left(X-X B_{i}\left(R_{i i}+B_{i}^{\top} X B_{i}\right)^{-1} B_{i}^{\top} X\right) \tilde{A}_{i}\right\} .
\end{aligned}
$$

Using part 2 of Subsection 3.5.2 in [18], we have the matrix inversion identity

$X-X W\left(Z+W^{\top} X W\right)^{-1} W^{\top} X=\left(X^{-1}+W Z^{-1} W^{\top}\right)^{-1}$, for any matrix $W$ and positive-definite matrices $X$ and $Z$. Therefore, for any $X>0$, we can rewrite $f(X)$ as

$$
\begin{aligned}
f(X)= & Q+\bar{A}^{\top}\left(X^{-1}+B R^{-1} B^{\top}\right)^{-1} \bar{A} \\
& +\sum_{i=1}^{N} \mathbb{E}\left\{\tilde{A}_{i}^{\top}\left(X^{-1}+B_{i} R_{i i}^{-1} B_{i}^{\top}\right)^{-1} \tilde{A}_{i}\right\} .
\end{aligned}
$$

Note that, if $X \geq Y \geq 0$, then

$$
\left(X^{-1}+W Z^{-1} W^{\top}\right)^{-1} \geq\left(Y^{-1}+W Z^{-1} W^{\top}\right)^{-1}
$$

for any matrix $W$ and positive-definite matrix $Z$. Therefore, if $X \geq Y \geq 0$, we get

$$
f(X) \geq f(Y)>0 .
$$

For any given $T \geq 0$, we define the sequence of matrices $\left\{X_{i}\right\}_{i=0}^{T}$ such that $X_{0}=0$ and $X_{i+1}=f\left(X_{i}\right)$. We have

$$
X_{1}=f\left(X_{0}\right)=f(0)=Q>0=X_{0} .
$$

Similarly, if we repeat this argument one more time, we get

$$
X_{2}=f\left(X_{1}\right) \geq f\left(X_{0}\right)=X_{1}>0 .
$$

The left-most inequity in (14) is true because $X_{1} \geq X_{0}$. We can repeat the same argument, and show that for all $1 \leq i \leq T-1$ that $X_{i+1} \geq X_{i}>0$. Using Theorem 3.1, we know that

$$
x_{0}^{\top} X_{T} x_{0}=\inf _{\{u(k)\}_{k=0}^{T-1}} J_{T}\left(x_{0},\{u(k)\}_{k=0}^{T-1}\right) .
$$

According to Theorem 5.1 in [11], since the system is mean square stabilizable the sequence $\left\{X_{i}\right\}_{i=0}^{\infty}$ is uniformly upperbounded; i.e., there exists $W \in \mathbb{R}^{n \times n}$ such that $X_{i} \leq W$ for all $i \geq 0$. Therefore, we get

$$
\lim _{T \rightarrow+\infty} X_{T}=X \in \mathbb{R}^{n \times n}
$$

since $\left\{X_{i}\right\}_{i=0}^{\infty}$ is an increasing upper-bounded sequence. In addition, we have $X>0$ since $X_{i}>0$ for all $i \geq 2$. Now, we need to prove that the limit $X$ in (15) is the unique positive definite solution of the modified discrete algebraic Riccati equation (12). This is done by a contrapositive argument. Assume that there exists $Z \in \mathcal{S}_{+}^{n}$ such that $f(Z)=Z$. For this matrix $Z$, we have

$$
Z=f(Z) \geq f(0)=X_{1}
$$

since $Z \geq 0$. Similarly, noting that $Z \geq X_{1}$, we get

$$
Z=f(Z) \geq f\left(X_{1}\right)=X_{2} \text {. }
$$




$$
\inf _{\{u(k)\}_{k=0}^{T-1}} J_{T}\left(x_{0},\{u(k)\}_{k=0}^{T-1}\right)=x_{0}^{\top} X_{T} x_{0} \leq x_{0}^{\top} Z x_{0}=\inf _{\{u(k)\}_{k=0}^{T-1}} \mathbb{E}\left\{x(T)^{\top} Z x(T)+\sum_{k=0}^{T-1} x(k)^{\top} Q x(k)+u(k)^{\top} R u(k)\right\}
$$

Repeating the same argument, we get $Z \geq X_{T}$ for all $T \geq 0$. Therefore, for each $T \geq 0$, we have the inequality in (16). Note that the right-most equality in (16) is a direct consequence of Theorem 3.1 and the fact that $Z=f(Z)=$ $f^{q}(Z)$ for any positive integer $q$. Let us define

$$
\left\{u^{*}(k)\right\}_{k=0}^{T-1}=\underset{\{u(k)\}_{k=0}^{T-1}}{\arg \inf } J_{T}\left(x_{0},\{u(k)\}_{k=0}^{T-1}\right),
$$

and $x^{*}(k)$ as the state of the system when the control sequence $u^{*}(k)$ is applied to it. Now, we get the inequality in (17) since, by definition, $\left\{u^{*}(k)\right\}_{k=0}^{T-1}$ is not the minimizer of this cost function. It is easy to see that, the right hand-side of (17) is equal to $J_{T}\left(x_{0},\left\{u^{*}(k)\right\}_{k=0}^{T-1}\right)+$ $\mathbb{E}\left\{x^{*}(T)^{\top} Z x^{*}(T)\right\}$. Thus, using (16) and (17), we get

$$
\begin{aligned}
x_{0}^{\top} X_{T} x_{0} & \leq x_{0}^{\top} Z x_{0} \\
& \leq J_{T}\left(x_{0},\left\{u^{*}(k)\right\}_{k=0}^{T-1}\right)+\mathbb{E}\left\{x^{*}(T)^{\top} Z x^{*}(T)\right\} \\
& =x_{0}^{\top} X_{T} x_{0}+\mathbb{E}\left\{x^{*}(T)^{\top} Z x^{*}(T)\right\} .
\end{aligned}
$$

Finally, thanks to the facts that $Q>0$ and

$$
\begin{array}{r}
\lim _{T \rightarrow+\infty} \mathbb{E}\left\{\sum_{k=0}^{T-1} x^{*}(k)^{\top} Q x^{*}(k)+u^{*}(k)^{\top} R u^{*}(k)\right\} \\
=\lim _{T \rightarrow+\infty} x_{0}^{\top} X_{T} x_{0}=x_{0}^{\top} X x_{0}<\infty,
\end{array}
$$

we get that $\lim _{T \rightarrow \infty} \mathbb{E}\left\{x^{*}(T)^{\top} x^{*}(T)\right\}=0$. Therefore, we have $\lim _{T \rightarrow \infty} \mathbb{E}\left\{x^{*}(T)^{\top} Z x^{*}(T)\right\}=0$. Taking limit form both sides of (18), when $T$ goes to infinity, results in $x_{0}^{\top} X x_{0}=x_{0}^{\top} Z x_{0}$ for all $x_{0} \in \mathbb{R}^{n}$. Thus, $X=Z$. This concludes the proof.

REMARK 3.2: Note that we can use the procedure introduced in the proof of Theorem 3.2 to numerically compute the unique positive-definite solution of the modified discrete algebraic Riccati equation in (12), that is, we can construct the sequence of matrices $\left\{X_{i}\right\}_{i=0}^{\infty}$, such that $X_{i+1}=f\left(X_{i}\right)$ with $X_{0}=0$ with $f(\cdot)$ as in (13). Because of (15), it is evident that, for each $\delta>0$, there exists a positive integer $q(\delta)$ such that $X_{q(\delta)}$ is in the $\delta$-neighborhood of the unique positive-definite solution of (12).

\section{Performance Degradation under Model INFORMATION LIMITATION}

In this section, we study the value of model information in control design using the closed-loop performance degradation caused by the lack of full model information in control design procedure. The performance degradation is captured using the ratio of the cost of the optimal controller with limited model information to the cost of the optimal controller with global plant model information (introduced in Appendix A).
Assume $\left\{u^{\mathrm{LMI}}(k)\right\}_{k=0}^{\infty}$ and $\left\{u^{\mathrm{FMI}}(k)\right\}_{k=0}^{\infty}$ denote the optimal controller with limited model information (introduced in Theorem 3.2) and the optimal controller with full model information (introduced in Proposition A.2), respectively. We define performance degradation ratio caused by the lack of full model information as

$$
r=\sup _{x_{0} \in \mathbb{R}^{n}} \frac{J_{\infty}\left(x_{0},\left\{u^{\mathrm{LMI}}(k)\right\}_{k=0}^{\infty}\right)}{J_{\infty}\left(x_{0},\left\{u^{\mathrm{FMI}}(k)\right\}_{k=0}^{\infty}\right)} .
$$

In order to find a reasonable upper-bound for this ratio, we make the following assumption:

Assumption 4.1: All subsystems are fully-actuated, that is, $B_{i i} \in \mathbb{R}^{n_{i} \times n_{i}}$ and $\underline{\sigma}\left(B_{i i}\right) \geq \epsilon>0$ for all $1 \leq i \leq N$, where $\underline{\sigma}(\cdot)$ denotes the smallest singular value of a matrix.

To simplify the presentation, we also assume that $Q=$ $R=I$. The next theorem presents an upper-bound for the performance degradation under Assumption 4.1.

TheOREM 4.1: Assume that the discrete-time linear stochastic system given in (1) satisfies Assumptions 3.1 and 4.1 , and is mean square stabilizable. The performance degradation ratio is upper-bounded by $r \leq 1+1 / \epsilon^{2}$.

Proof: Using the modified discrete algebraic Riccati equation (25) in Proposition A.2, the cost of the optimal control design with full model information $J_{\infty}\left(x_{0},\left\{u^{\mathrm{FMI}}(k)\right\}_{k=0}^{\infty}\right)=x_{0}^{\top} P^{\mathrm{FMI}} x_{0}$ is equal to

$$
\begin{array}{r}
x_{0}^{\top} P^{\mathrm{FMI}} x_{0}=x_{0}^{\top} Q x_{0}+x_{0}^{\top} \mathcal{R}\left(\bar{A}, P^{\mathrm{FMI}}, B, I\right) x_{0} \\
+\sum_{i=1}^{N} x_{0}^{\top} \mathbb{E}\left\{\mathcal{R}\left(\tilde{A}_{i}(k), P^{\mathrm{FMI}}, B, I\right)\right\} x_{0} .
\end{array}
$$

In addition, we know $P^{\mathrm{FMI}} \geq Q=I$, which (using the proof of Theorem 3.2) results in

$$
\begin{aligned}
\mathcal{R}\left(\bar{A}, P^{\mathrm{FMI}}, B, I\right) & \geq \mathcal{R}(\bar{A}, I, B, I), \\
\mathcal{R}\left(\tilde{A}_{i}(k), P^{\mathrm{FMI}}, B, I\right) & \geq \mathcal{R}\left(\tilde{A}_{i}(k), I, B, I\right) .
\end{aligned}
$$

Substituting (20)-(21) inside (19), we get

$$
\begin{aligned}
x_{0}^{\top} P^{\mathrm{FMI}} x_{0} \geq x_{0}^{\top}\left(I+\bar{A}^{\top}\left(I+B B^{T}\right)^{-1} \bar{A}\right) x_{0} \\
+\sum_{i=1}^{N} x_{0}^{\top} \mathbb{E}\left\{\tilde{A}_{i}(k)^{\top}\left(I+B_{i} B_{i}^{T}\right)^{-1} \tilde{A}_{i}(k)\right\} x_{0} .
\end{aligned}
$$

On the other hand, for a given $x_{0} \in \mathbb{R}^{n}$, the cost of the optimal control design with limited model information $J_{\infty}\left(x_{0},\left\{u^{\mathrm{LMI}}(k)\right\}_{k=0}^{\infty}\right)=x_{0}^{\top} P^{\mathrm{LMI}} x_{0}$ is upper-bounded by

$$
x_{0}^{\top} P^{\mathrm{LMI}} x_{0} \leq \mathbb{E}\left\{\sum_{k=0}^{+\infty} x(k)^{\top} x(k)+u(k)^{\top} u(k)\right\},
$$

where $u(k)=-B^{-1} A(k) x(k)$ and $x(k)$ is the state vector of the system when this control sequence is applied to the system. This is true since the deadbeat control design strategy 
$\inf _{\{u(k)\}_{k=0}^{T-1}} \mathbb{E}\left\{x(T)^{\top} Z x(T)+\sum_{k=0}^{T-1} x(k)^{\top} Q x(k)+u(k)^{\top} R u(k)\right\} \leq \mathbb{E}\left\{x^{*}(T)^{\top} Z x^{*}(T)+\sum_{k=0}^{T-1} x^{*}(k)^{\top} Q x^{*}(k)+u^{*}(k)^{\top} R u^{*}(k)\right\}$,

$u(k)=-B^{-1} A(k) x(k)$ uses only local model information for designing each subcontroller [7]. Therefore, we get

$$
\begin{aligned}
x_{0}^{\top} P^{\mathrm{LMI}} x_{0} \leq & \mathbb{E}\left\{x_{0}^{\top}\left(I+A(k)^{\top} B^{-\top} B^{-1} A(k)\right) x_{0}\right\} \\
= & x_{0}^{\top}\left(I+\bar{A}^{\top} B^{-\top} B^{-1} \bar{A}\right) x_{0} \\
& +x_{0}^{\top} \mathbb{E}\left\{\tilde{A}(k)^{\top} B^{-\top} B^{-1} \tilde{A}(k)\right\} x_{0} \\
= & x_{0}^{\top}\left(I+\bar{A}^{\top} B^{-\top} B^{-1} \bar{A}\right) x_{0} \\
& +\sum_{i=1}^{N} x_{0}^{\top} \mathbb{E}\left\{\tilde{A}_{i}(k)^{\top} B_{i i}^{-\top} B_{i i}^{-1} \tilde{A}_{i}(k)\right\} x_{0} .
\end{aligned}
$$

The second equality is a direct result of the assumption that the subsystems are stochastically independent of each other. Let us define the set $\mathcal{M}=\{\bar{\beta} \mid r \leq \bar{\beta}\}$. If a real number $\beta$ satisfy $\beta P^{\mathrm{FMI}}-P^{\mathrm{LMI}} \geq 0$, then $\beta \in \mathcal{M}$. We have

$$
\begin{aligned}
& \beta P^{\mathrm{FMI}}-P^{\mathrm{LMI}} \geq(\beta-1) I \\
& +\bar{A}^{\top}\left[\beta\left(I+B B^{\top}\right)^{-1}-B^{-\top} B^{-1}\right] \bar{A} \\
& +\sum_{i=1}^{N} \mathbb{E}\left\{\tilde{A}_{i}(k)^{\top}\left[\beta\left(I+B_{i} B_{i}^{\top}\right)^{-1}-B_{i i}^{-\top} B_{i i}^{-1}\right] \tilde{A}_{i}(k)\right\} .
\end{aligned}
$$

Therefore, a sufficient condition for $\beta P^{\mathrm{FMI}}-P^{\mathrm{LMI}} \geq 0$ is

$$
\begin{aligned}
& (\beta-1) I+\bar{A}^{\top}\left[\beta\left(I+B B^{\top}\right)^{-1}-B^{-\top} B^{-1}\right] \bar{A} \\
& +\sum_{i=1}^{N} \mathbb{E}\left\{\tilde{A}_{i}(k)^{\top}\left[\beta\left(I+B_{i} B_{i}^{\top}\right)^{-1}-B_{i i}^{-\top} B_{i i}^{-1}\right] \tilde{A}_{i}(k)\right\} \geq 0 .
\end{aligned}
$$

As a result, we get $\left[1+1 / \epsilon^{2},+\infty\right) \subseteq \mathcal{M}$ since $\underline{\sigma}(B) \geq \epsilon$. This shows that

$$
r=\sup _{x_{0} \in \mathbb{R}^{n}} \frac{x_{0}^{\top} P^{\mathrm{LMI}} x_{0}}{x_{0}^{\top} P^{\mathrm{FMI}} x_{0}} \leq 1+\frac{1}{\epsilon^{2}} .
$$

This concludes the proof.

REMARK 4.1: Assuming that the system satisfies Assumption 4.1, when the variances of the plant model parameters tend to infinity, the optimal controller with limited model information (introduced in Theorem 3.2) approaches deadbeat. Therefore, when our belief about other subsystems is inaccurate, we simply cannot risk using their statistical information and as a result the deadbeat is the best controller (since it discards this information). Therefore, in this case, the upper-bound presented in Theorem 4.1 becomes tight.

\section{Numerical EXAMPLE}

Consider a simple linear discrete-time dynamical system composed of two scalar subsystems as

$$
\left[\begin{array}{l}
x_{1}(k+1) \\
x_{2}(k+1)
\end{array}\right]=\left[\begin{array}{ll}
a_{11}(k) & a_{12}(k) \\
a_{21}(k) & a_{22}(k)
\end{array}\right]\left[\begin{array}{l}
x_{1}(k) \\
x_{2}(k)
\end{array}\right]+\left[\begin{array}{cc}
1 & 0 \\
0 & 1
\end{array}\right]\left[\begin{array}{l}
u_{1}(k) \\
u_{2}(k)
\end{array}\right]
$$

where $x_{i}(k) \in \mathbb{R}$ and $u_{i}(k) \in \mathbb{R}$ are the state and the control input of subsystem $i$, respectively. Let us assume that

$$
\mathbb{E}\left\{a_{11}\right\}=2.0 \text { and } \mathbb{E}\left\{\left(a_{11}-\mathbb{E}\left\{a_{11}\right\}\right)^{2}\right\}=0.4,
$$

$$
\begin{gathered}
\mathbb{E}\left\{a_{12}\right\}=1.0 \text { and } \mathbb{E}\left\{\left(a_{12}-\mathbb{E}\left\{a_{12}\right\}\right)^{2}\right\}=0.1, \\
\mathbb{E}\left\{\left(a_{11}-\mathbb{E}\left\{a_{11}\right\}\right)\left(a_{12}-\mathbb{E}\left\{a_{12}\right\}\right)\right\}=0.1,
\end{gathered}
$$

and

$$
\begin{gathered}
\mathbb{E}\left\{a_{22}\right\}=3.0 \text { and } \mathbb{E}\left\{\left(a_{22}-\mathbb{E}\left\{a_{22}\right\}\right)^{2}\right\}=0.2, \\
\mathbb{E}\left\{a_{12}\right\}=1.0 \text { and } \mathbb{E}\left\{\left(a_{12}-\mathbb{E}\left\{a_{21}\right\}\right)^{2}\right\}=0.1, \\
\mathbb{E}\left\{\left(a_{21}-\mathbb{E}\left\{a_{21}\right\}\right)\left(a_{22}-\mathbb{E}\left\{a_{22}\right\}\right)\right\}=0.1 .
\end{gathered}
$$

The goal is to optimize the following performance criterion

$$
J=\sum_{k=1}^{\infty}\left[\begin{array}{l}
x_{1}(k) \\
x_{2}(k)
\end{array}\right]^{\top}\left[\begin{array}{l}
x_{1}(k) \\
x_{2}(k)
\end{array}\right]+\left[\begin{array}{l}
u_{1}(k) \\
u_{2}(k)
\end{array}\right]^{\top}\left[\begin{array}{l}
u_{1}(k) \\
u_{2}(k)
\end{array}\right] .
$$

1) Optimal Controller Using Statistical Model Information: This optimal controller is derived in [11]. Using Theorem 5.2 from [11], we get

$$
P^{\mathrm{SMI}}=\left[\begin{array}{cc}
11.8923 & 7.5185 \\
7.5185 & 14.4816
\end{array}\right]
$$

which results in the optimal controller

$$
u^{\mathrm{SMI}}(k)=\left[\begin{array}{ll}
-1.8361 & -1.0494 \\
-1.0150 & -2.7822
\end{array}\right]\left[\begin{array}{l}
x_{1}(k) \\
x_{2}(k)
\end{array}\right] .
$$

2) Optimal Controller Using Full Model Information: This optimal controller is derived in Appendix A. Using Proposition A.2, we get

$$
P^{\mathrm{FMI}}=\left[\begin{array}{cc}
5.7805 & 5.0098 \\
5.0098 & 10.4446
\end{array}\right],
$$

which results in the optimal controller

$$
u^{\mathrm{FMI}}(k)=K^{\mathrm{FMI}}\left[\begin{array}{l}
x_{1}(k) \\
x_{2}(k)
\end{array}\right],
$$

where

$$
K^{\mathrm{FMI}}=\left[\begin{array}{ll}
-0.7820 & -0.0954 \\
-0.0954 & -0.8709
\end{array}\right]\left[\begin{array}{ll}
a_{11}(k) & a_{12}(k) \\
a_{21}(k) & a_{22}(k)
\end{array}\right] .
$$

3) Optimal Controller Using Limited Model Information: This optimal controller is derived in Section III. Using Theorem 3.2, we have

$$
P^{\mathrm{LMI}}=\left[\begin{array}{cc}
5.8170 & 5.0212 \\
5.0212 & 10.4612
\end{array}\right],
$$

which results in the optimal controller

$$
u^{\mathrm{LMI}}(k)=K^{\mathrm{LMI}}\left[\begin{array}{l}
x_{1}(k) \\
x_{2}(k)
\end{array}\right]
$$

where

$$
K^{\mathrm{LMI}}=\left[\begin{array}{ll}
-0.8533 a_{11}(k)+0.0449 & -0.8533 a_{12}(k)-0.2148 \\
-0.9127 a_{21}(k)-0.1482 & -0.9127 a_{22}(k)+0.0298
\end{array}\right] .
$$


It is easy to see that $P^{\mathrm{FMI}} \leq P^{\mathrm{LMI}} \leq P^{\mathrm{SMI}}$. In addition, one can check that

$$
r=\sup _{x_{0} \in \mathbb{R}^{n}} \frac{x_{0}^{\top} P^{\mathrm{LMI}} x_{0}}{x_{0}^{\top} P^{\mathrm{FMI}} x_{0}}=1.0088 \leq 1+1 / \epsilon^{2}=2,
$$

since, in this example, $\epsilon=1$. This shows that the optimal controller with limited model information is at most only $1 \%$ worse than the optimal controller with full model information. It is interesting to note that, with only access to precise local model information, in this numerical example, one can expect a huge improvement in the closed-loop performance in comparison to the optimal controller with only statistical model information

$$
\sup _{x_{0} \in \mathbb{R}^{n}} \frac{x_{0}^{\top} P^{\mathrm{SMI}} x_{0}}{x_{0}^{\top} P^{\mathrm{LMI}} x_{0}}=2.3607 \text {. }
$$

\section{CONCLUSION}

We presented a statistical framework for the study of control design under limited model information. We found the best performance achievable by a limited model information control design method. We also studied the value of information in control design using the performance degradation ratio. Possible future work will focus on generalizing the results to discrete-time Markovian jump linear systems and to decentralized controllers.

\section{ACKNOWLEDGEMENT}

The authors would like to thank Cédric Langbort for invaluable discussions and suggestions.

\section{REFERENCES}

[1] D. Swaroop and J. K. Hedrick, "Constant spacing strategies for platooning in automated highway systems," Journal of Dynamic Systems, Measurement, and Control, vol. 121, no. 3, pp. 462-470, 1999.

[2] S. Massoud Amin and B. Wollenberg, "Toward a smart grid: power delivery for the 21st century," IEEE Power and Energy Magazine, vol. 3, no. 5, pp. $34-41,2005$.

[3] R. R. Negenborn, Z. Lukszo, and H. Hellendoorn, eds., Intelligent Infrastructures, vol. 42. Springer, 2010.

[4] K. Loparo and G. Blankenship, "A probabilistic mechanism for small disturbance instabilities in electric power systems," IEEE Transactions on Circuits and Systems, vol. 32, no. 2, pp. 177 - 184, 1985.

[5] M. Brucoli, M. L. Scala, F. Torelli, and M. Trovato, "A semi-dynamic approach to the voltage stability analysis of interconnected power networks with random loads," International Journal of Electrical Power \& Energy Systems, vol. 12, no. 1, pp. 9-16, 1990.

[6] F. Wu and C.-C. Liu, "Characterization of power system small disturbance stability with models incorporating voltage variation," IEEE Transactions on Circuits and Systems, vol. 33, no. 4, pp. 406 - 417, 1986.

[7] F. Farokhi, "Decentralized control design with limited plant model information," Licentiate Thesis, 2012. http://urn.kb.se/ resolve?urn=urn:nbn: se:kth: diva-63858.

[8] C. Langbort and J. Delvenne, "Distributed design methods for linear quadratic control and their limitations," IEEE Transactions on Automatic Control, vol. 55, no. 9, pp. 2085 -2093, 2010.

[9] F. Farokhi, C. Langbort, and K. Johansson, "Control design with limited model information," in Proceedings of the American Control Conference, pp. 4697 -4704, 2011.

[10] F. Farokhi, C. Langbort, and K. Johansson, "Optimal disturbanceaccommodation design with limited model information," in Proceedings of the American Control Conference, pp. 4757-4764, 2012.

[11] W. De Koning, "Infinite horizon optimal control of linear discrete time systems with stochastic parameters," Automatica, vol. 18, no. 4, pp. $443-453,1982$.
[12] W. De Koning, "Optimal estimation of linear discrete-time systems with stochastic parameters," Automatica, vol. 20, no. 1, pp. 113 115, 1984.

[13] M. Aoki, Optimization of stochastic systems: topics in discrete-time systems. Academic Press, 1967.

[14] A. R. Tiedemann and W. L. De Koning, "The equivalent discrete-time optimal control problem for continuous-time systems with stochastic parameters," International Journal of Control, vol. 40, no. 3, pp. 449466, 1984.

[15] O. C. Imer, S. Yüksel, and T. Basar, "Optimal control of LTI systems over unreliable communication links," Automatica, vol. 42, no. 9, pp. 1429 - 1439, 2006.

[16] J. Swigart and S. Lall, "An explicit dynamic programming solution for a decentralized two-player optimal linear-quadratic regulator," in Proceedings of mathematical theory of networks and systems, 2010.

[17] T. J. A. Wagenaar and W. L. De Koning, "Stability and stabilizability of chemical reactors modelled with stochastic parameters," International Journal of Control, vol. 49, no. 1, pp. 33-44, 1989.

[18] H. Lütkepohl, Handbook of matrices. Wiley, 1996.

\section{APPENDIX A}

\section{Control Design with Full Model Information}

In this appendix, we assume that, when designing subcontroller $i$, we have access to the full model information, that is, we can observe the entire model parameters $\left\{A_{i j}(k) \mid 1 \leq\right.$ $i, j \leq N\}$ when designing each local controller. The following proposition gives the solution to the finite-horizon optimal control problem.

Proposition A.1: The solution of the finite horizon optimal control problem is given by

$$
\begin{aligned}
u(k)=-\left(R+B(k)^{\top}\right. & P(k+1) B(k))^{-1} \\
& \times B(k)^{\top} P(k+1) A(k) x(k),
\end{aligned}
$$

where $\{P(k)\}_{k=0}^{T}$ can be found using the backward difference equation

$$
\begin{aligned}
P(k)= & Q(k)+\mathcal{R}(\bar{A}(k), P(k+1), B(k), R) \\
& +\sum_{i=1}^{N} \mathbb{E}\left\{\mathcal{R}\left(\tilde{A}_{i}(k), P(k+1), B(k), R\right)\right\},
\end{aligned}
$$

with the boundary condition $P(T)=Q(T)$.

Proof: The proof is similar to the proof of Theorem 3.1.

This result can also be extended to infinite-horizon cost function using the next proposition.

Proposition A.2: Assume that the discrete-time linear stochastic system given in (1) satisfies Assumption 3.1 and is mean square stabilizable. The solution of the infinite-horizon optimal control problem is given by

$$
u(k)=-\left(R+B^{\top} P B\right)^{-1} B^{\top} P A(k) x(k),
$$

where $P$ is the unique finite positive-definite solution of the modified discrete algebraic Riccati equation

$$
P=Q+\mathcal{R}(\bar{A}, P, B, R)+\sum_{i=1}^{N} \mathbb{E}\left\{\mathcal{R}\left(\tilde{A}_{i}(k), P, B, R\right)\right\} .
$$

Furthermore, this controller mean square stabilizes the system and

$$
J_{\infty}\left(x_{0},\{u(k)\}_{k=0}^{\infty}\right)=x_{0}^{\top} P x_{0} .
$$

Proof: The proof is similar to the proof of Theorem 3.2. 